\title{
VICTORIA SURLIUGA
}

\section{LA GALERLA DI G.B. MARINO TRA PITTURA E POESIA}

Ha voluto adunque, ha saputo, ed è stato sempre solito Iddio di dipingere, e (sì come nella sua sacra cosmopea canta il gran cronista Mosè) ha nella creazione dell'universo varie e diverse maraviglie dipinte.

G.B. Marino, Diceria prima: la Pittura.

La Galeria di G.B. Marino rappresenta un caso paradigmatico di utilizzazione del concetto di ut pictura poesis nelle prime fasi dell'epoca barocca. Nel sedicesimo secolo, non solo pittura e poesia si trovarono unificate, ma si teorizzò anche che ciò che legava le arti nobili e quelle della memoria fosse la loro natura figurale. Ad esempio, nel 1555, Giovanni Pietro Capriano parlava di poesia, pittura e scultura come delle più perfette tra le arti nobili. D'altro canto, nel 1572, Lodovico Castelvetro, facendo riferimento alle arti commemorative della memoria, riproponeva una contrapposizione tra arti e artigianato. La loro funzionalità riguardava per le arti la preservazione della memoria, e per l'artigianato la quotidianità, dunque i bisogni immediati. Fu in questo periodo che il motto oraziano ut pictura poesis venne invertito in ut poesis pictura, dando così voce alla convinzione che fosse l'elemento poetico a separare la pittura dall'artigianato. Il detto attribuito da Simonide di Ceo a Plutarco nel De Gloria Atheniensium, ovvero che la pittura è una poesia muta, e quest'ultima una pittura parlante, divenne di gran voga in conseguenza della discussione sul precetto dell' Ars poetica oraziana, cioè "com'è la pittura, tale è la poesia", sebbene il concetto non fosse inteso da Orazio in modo così normativo come poi venne interpretato dai sostenitori della teoria umanistica della pittura. Sostiene l'Ars Poetica:

Ut pictura poesis: erit quae si propius stet te capiat magis, et quaedam si longius abstes. Haec amat obscurum, volet haec sub luce videri, Iudicis argutum quae non formidat acumen; Haec placuit semel, haec decies repetita placebit ${ }^{1}$

[Come la pittura cosi la poesia: quello al quale starai più vicino 
ti prende di più, e quell'altro se ne stai più lontano.

Questo ama l'oscurità mentre quest'altro vuole

esser visto in piena luce, reggendo il giudizio acuto del critico;

Questo ti piacque una volta, quest'altro ti piacerà anche se visto dieci volte].

Orazio attribuiva lo stesso diritto di immaginazione sia ai poeti che ai pittori, limitandolo però al congruo e al probabile. La sua comparazione tra poesia e pittura auspicava soprattutto una flessibilità di giudizio critico nel dichiarare che la poesia si dovesse paragonare alla pittura. Quest'ultima non richiede semplicemente uno stile dettagliato, tale da poter essere analizzato attentamente, ma anche un'organizzazione formale di più ampia portata, capace di soddisfare anche chi guarda il dipinto da una certa distanza. Orazio non propugnava dunque una teoria delle arti sorelle e, anzi, secondo Massimo Verdicchio, voleva invece indicare

...che nella pittura come nella poesia ci sono buone e cattive opere, opere che viste alla luce possono sempre piacere ed altre che sebbene protette dall'ombra piacciono solo una volta. É nel ' 400 e nel ' 500 che viene fatta la confusione con l'alterazione della punteggiatura. I due punti (:) dopo 'ut pictura poesis' vengono eliminati cambiando il tono della frase dal più debole senso di 'talvolta sarà il caso che' al più forte $\mathrm{e}$ categorico 'un poema sarà come una pittura' (Verdicchio 775).

Uno dei motivi per cui l'Ars poetica oraziana venne interpretata nel modo normativo a cui si è accennato era che si voleva dimostrare come la pittura fosse a pieno titolo un'arte liberale proprio in virtù della sua rassomiglianza con la poesia. In questo contesto, anche Mario Praz ha ribadito come l'espressione ut pictura poesis, nel significato originale oraziano, avesse implicazioni diverse da come venne poi interpretata, e soprattutto meno ambiziose. Il poeta voleva solamente intendere che alcuni dipinti, come certe poesie, piacciono una volta sola, mentre altri possono essere interpretati criticamente più volte. Il che confermerebbe il commento di Simonide di Ceo: la pittura è muta poesia e la poesia, invece, una pittura parlante.

Per diversi scrittori, la qualità figurativa, o metaforica, era la caratteristica principale delle arti nobili e non artigianali. In particolare per Emanuele Tesauro, nel Cannoccliale Aristotelico, la metafora e il "parlar figurato" in genere, e quindi l'aspetto figurale del linguaggio, sono la caratteristica dominante e unica dell'oratoria e della poesia. L'opera di Tesauro, con la sua teorizzazione della metafora come generatrice del linguaggio, rappresenta per molti versi il culmine della poetica barocca, secondo la cui estetica la poesia fornisce il soggetto alla pittura, da cui poi viene illustrata (Tatarkiewicz 115). 
La Galeria si trova nel punto di convergenza del dibattito che accomuna pittura e poesia. Pubblicata per la prima volta a Venezia per le edizioni Ciotti nell'autunno del 1619, alla prima edizione ne segui subito un'altra nel 1620 , e poi circa sedici altre tra il 1620 e il 1675 . Originariamente, il volume doveva apparire illustrato da immagini sontuose, come Narino aveva infatti scritto nell'introduzione, intitolata $A$ chi legge: "quando [la Galeria] uscirà poi istoriata ed ornata di figure, poi che non si è potuto al tutto supplire in una rolta" (Marino 1979, 3). Invece, nonostante le grandi aspettative, l'opera finì per non essere arricchita da nessun apparato iconografico. Tutto lo sforzo di Marino si concentrò sulla pubblicazione dei versi, aggiungendo alle successive edizioni, da lui curate, poesie su opere che aveva acquistato per la sua collezione, e altre basate su dipinti e statue immaginarie di figure illustri del passato o a lui contemporanee.

La Galeria è difficilmente classificabile, sia per la molteplicità delle intenzioni di Marino che per la varietà dei materiali utilizzati, i quali danno vita a un capriccioso insieme di poesie iconografiche che si inseriscono di forza nel solco della tradizione della corrispondenza tra arti sorelle. Come ha osservato Alessandro Martini, "cadrà l'ambizioso progetto del raffronto sulle pagine a stampa fra disegno e poesia, ma nei versi ricorrerà sempre questo doppio elemento: la lode e lo scherzo, nel quale propriamente consiste il capriccio" (Martini 658). Nella Galeria, però, Marino pone anche le basi della questione dell'ekphrasis, o descrizione in parole di un'opera pittorica, a partire da un "gemellaggio" tra pittura e poesia da lui dato per scontato (almeno a quanto sostiene Golahny). Per Marino, il linguaggio scritto può incorporare quello visivo, e il poeta napoletano risolve il problema di come la poesia legga le arti. Si ha ekphrasis quando la parola scritta è per il lettore un riferimento a delle immagini, quando vi è rappresentazione verbale del segno iconico. L'immagine a cui il testo rimanda può esistere o meno, ma perché vi sia ekphrasis bisogna che il testo scritto vi faccia riferimento e che quindi la composizione poetica abbia delle opere d'arte come soggetto. Nella Galeria, l'immagine che conta è quella creata dall'intelletto e successivamente trasferita in versi, soprattutto nel caso di liriche riguardanti opere d'arte inventate da Marino, e che ribadiscono quindi la superiorità, per il poeta napoletano, della poesia sulla pittura. Francesco Guardiani, a questo proposito, mette in guardia dal confondere "immagine" con "figura" (o "figurazione"); è la raffigurazione mentale ad importare a Marino, per cui

...l'immagine che sta veramente a cuore a Marino non è quella della figura chiusa permanentemente sulla tela, ma quella che sta a monte della realizzazione pittorica, è la figura evanescente tracciata dallintelletto (Guardiani 1988, 650)². 
A una prima lettura, ciò che balza agli occhi nella Galeria è un'apparente mancanza di unità di intenti e di finalità. Guardiani mette in evidenza che "il problema della cementazione delle varie parti di un'opera che si vuole unita al momento dell'edizione seppure nel corso della stesura mostra per l'unità un palese disprezzo o, quanto meno, una totale noncuranza" (Guardiani 1988, 647). L'intenzionalità originaria dell'opera stava nell'essere una specie di passeggiata tra le opere d'arte che il primo mecenate di Marino, il principe di Conca, teneva nella galleria del suo palazzo. Infatti lo stesso poeta scrive, nella Diceria sulla pittura, riferendosi al palazzo del principe:

Entra, entra uomo, ne' penetrali di questo portico stupendo, spazia entro i ricessi di questo bel teatro, considera le prospettive, gli artifici e le maraviglie di questa divina architettura, se vuoi stupire (Marino 1960, 120-21, corsivo nostro).

Si può dire che la Galeria non segue certi precetti aristotelici, quali le regole dell'unità temporale, ma rispetta, in un certo senso, una regola di unità spaziale, realizzata attraverso una sequenza di immagini artistiche colte in un'ipotetica passeggiata tra pitture e statue. Lo stesso Guardiani, pur rimarcando la frammentarietà degli elementi della Galeria, sostiene tuttavia che vi sia presente un elemento che "salda nell'unico corpo della Galeria i suoi oltre seicento membri indipendenti, nel loro individuale delinearsi intorno ad un concetto ed alla forma che esso produce" (Guardiani 1988, 648). Nello stesso articolo, infatti, Guardiani osserva come, nella serie di oggetti della Galeria, "si voglia offrire... l'insieme stesso della creazione artistica" (Guardiani 1988, 649).

Nella già citata introduzione alla Galeria, ovvero la parte intitolata $A$ cbi legge, Marino espone lo scopo dell'opera nei seguenti termini, quasi come uno "scherzo" intorno a poche opere d'arte, tra le moltissime di cui si potrebbe parlare:

L'intenzione principale dell'Autore non è stata di comporre un Museo universale sopra tutte le materie che possono essere rappresentate dalla Pittura e dalla Scultura, ma di scherzare intorno ad alcune poche, secondo i motivi Poetici che alla giornata gli son venuti in fantasia. Né di fare Elogii distinti a tutti coloro che sono degni di loda, ma di celebrare gli uomini più illustri dell'età antica, de' moderni solamente morti, e de' vivi appena alcuni Prencipi da lui domesticamente conosciuti, ed alquanti suoi cari e particolari amici, i quali per avere esposte le loro fatiche alla pubblica luce, sono noti per fama, e le cui imagini gli sono state in effetto da essi medesimi donate (Marino 1979, 3). 
La Galeria è da considerarsi soprattutto come il manifesto della passione di Marino per le arti e per chi le pratica. Il coinvolgimento di Marino, poeta, con la pittura e il suo mondo, è ben noto. Si sa che il poeta aveva aiutato molto Nicolas Poussin all'inizio della sua carriera, e che il pittore, ancora giovane, aveva trovato nel poeta napoletano un prezioso sostenitore $^{3}$. Inoltre, Marino era anche un collezionista e raccoglieva dipinti e disegni che si faceva regalare dagli artisti a lui contemporanei, spesso in cambio dell'inserimento nella sua Galeria di una pocsia a loro dedicata. La Galeria, con il tempo, prese così sempre più la forma di una raccolta di poesie unitarie per argomento anche se casuali nella loro concatenazione. Secondo Marziano Guglielminetti, il poeta napoletano voleva soprattutto creare, sia attraverso la sua raccolta di opere d'arte che tramite le loro rappresentazioni verbali, "una collezione di disegni e di stampe così ricca, da emulare quella di alcuni suoi mecenati, quali il principe di Conca ed il cardinal Doria" (Guglielminetti 17). La prima parte della Galeria era stata dedicata da Marino proprio al Signor Giovan Carlo Doria, e la seconda al Signor Luigi Centurioni Marchese di Morsasco. Spesso il poeta chiedeva molto apertamente che i pittori gli facessero dono di disegni o dipinti; una simile richiesta si può leggere ad esempio nel madrigale I enere assisa in una conca di Bernardo Castello:

Oh come in vaga conca

siede lieta e vezzosa

la bella Dea che 'nsanguinò la rosa!

La tua mercé, Castello, io la vagheggio

senza alcun'ombra o velo

più bella in mar, che ' $\mathrm{n}$ Cielo,

anzi fatta immortale anco la veggio

piì nella tela tua,

che nella sfera sua (Marino 1979, 11, corsivo nostro).

E si veda anche la lettera di Marino a Bernardo Castello, in cui il poeta scrive: "Signor Bernardo mio carissimo, credami V.S. ... che in questa mia indisposizione non ho altro consolamento e refrigerio maggiore che la I enere di V.S., la quale mi ho fatta porre a riscontro del letto e tutto il di la vagheggio". Inoltre, a proposito della passione di Marino per il collezionismo di opere d'arte, la stessa lettera prosegue così: "se il bello e "l bene è per se stesso naturalmente comunicabile, di questa bellezza non di meno voglio io esser tirannicamente solo il goditore" (Marino 1911, 44).

Presa complessivamente, la Galeria è una raccolta di 453 componimenti divisi tra Pitture e Sculture. Molte si basano su opere d'arte ispirate da scritti ovidiani e biblici. Altre, come si è detto, fanno riferimento ad opere 
d'arte immaginarie. Le Pitture si dividono in Favole, Historie, Ritratti, Capricci, e con questi ultimi si intendono poesie dedicate quasi tutte a dipinti o disegni di gusto miniaturistico. Le Favole descrivono opere d'arte con soggetti mitologici e le Istorie si riferiscono a temi biblici, tratti tanto dal Vecchio quanto dal Nuovo Testamento. Carracci, Guido Reni, Caravaggio, Bronzino, Raffaello, Correggio e Parmigianino sono alcuni dei pittori le cui opere sono descritte nelle Favole e nelle Istorie. Martini, nel descrivere le caratteristiche di queste farole, storie, e ritratti, fa riferimento a una contiguità di sacro e di profano in cui

si apre una prospettiva talmente fitta di rinvii letterari, [in cui] sta una così intricata e spesso dissacrante intertestualità da escludere i rinvii pittorici che si offrono quasi spontaneamente al non addetto ai lavori e che sembrano imporsi solo là dove la nostra meno perdonabile ignoranza della traduzione letteraria non ci suggerisce più stringenti addentellati (Martini, 656).

Una definizione di quello che si intende con il termine Galeria e una descrizione dell'opera, espressa con le parole stesse del Marino, si trova fin dall'introduzione del 1614 alla terza parte delle Rime, nella quale Marino anticipa quale sarà la struttura dell'opera che deve ancora completare:

Havvi la Galeria, ch'è come dir Pinacoteca, luogo dove anticamente (come riferisce Petronio Arbitro) si conservano le pitture. Ed a questa gli diede qualche occasione Filostrato con le sue Imagini, se ben egli si è allontanato assai dalla sua via. È divisa in due parti, cioè Pitture e Sculture, e sono ambedue compartite in Farole, Istorie, e Ritratti. L'Istorie sono sacre e profane, e vengono spiegate con varie fantasie poetiche e con le lodi de' maestri più famosi, secondo l'occasione che ne porgono molte figure di lor mano. Le Favole sono le più notabili cavate da' poeti greci e latini. E questa parte ha da stamparsi poi istoriata con intaglio di bellissimi disegni clal Cavaliere accumulati, opera d'artefici eccellenti. Tra i Ritratti entrano in simulacri di diversi uomini illustri sí in armi come in lettere, tanto moderni quanto antichi, talché formano a guisa di un Musero; e sopra ciascuna imagine si scherza con qualche bizaria secondo le azioni del rapresentato, seguitando in ciò lo stile che tennero tra' latini Fausto Sabeo e Giulio Cesare Scaligero, che ne lasciarono molti epigrammi, e tra' volgari I'Unico Accolti e 'I conte Baldassar Castiglioni, de' quale se ne veggono alcune poche ottave (cit. da Pieri in Marino 1979, XXX-I).

Questo scritto introduttivo venne attribuito da Marino a un gentiluomo torinese ma si presume che fosse stato redatto dal poeta stesso, che riusciva cosi a informare il lettore delle fonti letterarie da lui usate come modelli per la sua ispirazione. In genere, Marino tendeva ad usare fonti 
poco recenti rispetto alla sua epoca, e quindi difficilmente identificabili, ma in questo caso non sta citando poeti ma critici e prosatori, c forse per questo è più portato a dichiarare la loro influenza.

Il termine Galeria, sostiene Carlo Dionisotti, "come titolo di un'opera di poesia, ancora era ai primi del Seicento rara e nuova in lingua italia, e suonava, come di fatto era, straniera" (Dionisotti 482, ma si reda anche Besomi). Oltre che a rappresentare la descrizione di un museo ideale, scrive Eugenia Paulicelli, la Galeria era anche un esempio di teatro della memoria:

La Galeria evoca sin dal titolo la nozione di spazio architettonico del testo, infatti lo stesso autore lo definisce un "museo" dove conservare/ eternare una serie di personaggi della storia della letteratura, della pittura, dell'antichità classica ... [e] si ha la netta impressione ... di entrare e varcare vere e proprie stanze ... È la scansione di questi spazi e loci a rendere la Galeria più che un museo, un piccolo teatro della memoria, che agisce come riorganizzazione e riuso dei materiali, dalla letteratura alle arti visive, dalle fonti classiche a quelle bibliche ... nell'intento di superare i limiti stessi della poesia e della parola andando a frugare nei tesori nascosti degli scrigni di pittori e scultori (Paulicelli 256-7).

La passeggiata ideale di Marino, tra logge e androni, nonché tra una serie e l'altra di quadri, diventa così una catalogazione di pitture che intendono ricoprire ogni possibile spazio visivo. Nel suo insieme, l'opera si avvicina cosi al genere pittorico cosiddetto galleristico, in cui il soggetto di un dipinto è appunto una galleria o uno scorcio di architettura in prospettiva. Si tratta, come ha osservato Martini, di veri e propri "scambi di ruolo" fra Arte e Natura, vero e finto, moto e stasi, parola e silenzio:

...negli specchiamenti e nelle gare fra Pittura e Poesia, penna e pennello, è certo che il capriccio mariniano è essenzialmente di natura metaforica, si specializza come arguzia ingegnosa, tal quale sarà riproposta e codificata nel Cannocchiale aristotelico del Tesauro (Martini 658).

In questo insieme di metafore argute, c'è naturalmente ampio spazio riservato alla meraviglia, sia quella del poeta nel descrivere i quadri, sia quella che dovrebbe essere suscitata nel lettore dalla lettura delle descrizioni poetiche (si vedano i commenti di Warman). Ed è per questo che Jean Hagstrum, paragonando la Galerio del Marino alla galleria di pittori del poeta inglese Marvell, si lamenta del fatto che nel poeta italiano non vi sia spazio per la contemplazione dell'animat. Per questo motivo, non stupisce il tono ancora più critico del commento di Marzio Pieri nella sua introduzione ai due volumi della Galeria: 
La Galeria pare richiudersi su se stessa, sulla propria materialità letteraria. Delle pitture, cosi, potrebbe parlare un cieco. Insieme, lo spazio del sonetto o del predominante madrigale diviene, propriamente, letteralmente, spazio (spiazzo), area, campo di gioco, o meglio scacchiere o tavoliere offerto agli studiosi, movimenti, au ralenti, d'una variantistica poco aerata, tendenzialmente monotematica. A quel modo che l'ingombro catalogo degli uomini e delle donne celebri è rigorosamente contemplato a porte chiuse, finestre chiuse, che l'aria o la luce, affrontando quei manichini di parole, non li scancellino. Qui, nei «ritratti», la favola o l'istoria che offriva il destro alla macchinetta poetica per le «pitture», è sostituita dall'aneddoto ... quando non da una sorta di indice biografico in versi, nemmeno sempre appoggiati, aneddoto o indice, a una soluzione acuminata (Marino 1979, XLI).

La Galeria è, sempre secondo Pieri, composta di due opere distinte, di cui una legata alle arti figurative e, l'altra, una specie di enciclopedia, costituita dalla sezione dei Ritratti. Questi sono per la maggior parte immaginari e sono una serie di commenti su persone famose o note per la loro infamia fin da tempi remoti. Marino include anche poesie su ritratti di se stesso (durante la sua vita, infatti, il poeta venne ritratto da Caravaggio, Domenichino, e Reni, e da vari pittori minori). I Ritratti sono suddivisi in una lunga e precisa serie di categorie umane e sociali quali, ad esempio, quelli degli Uomini, ovvero: Prencipi, Capitani ed Heroi; Tiranni, Corsari, e Scelerati; Pontefici e Cardinali; Padri Santi e Theologi; Negromanti ed Heretici; Oratori e Predicatori; Filosofi ed Humanisti; Historici; Giuriconsulti e Medici; Matematici e Astrologi; Poeti Greci; Poeti Latini; Poeti Volgari; Pittori e Scultori; Ritratti di diversi Signori e Letterati amici dell'Autore; Ritratti Burleschi. C'è poi una categoria di Ritratti di Donne, che include: Belle Caste e Magnanime; Belle Impudiche e Scelerate; Bellicose e Virtuose; e seguono sette Caprici. Infine, c'è la parte intitolata Le Sculture, che comprende: Statue; Rilievi, Modelli e Medaglie; e altri dodici Caprici. Vi sono componimenti sulle statue di Michelangelo e un gruppo di poesie su statue fatte di materiali eterogenei come la neve, l'argento, lo zucchero, la cera e l'ambra. Diverse categorie includono solo poche poesie, e la rigorosità di queste classificazioni, fatte a seconda del soggetto trattato, dimostra che Marino voleva dimostrare di essere in grado di scrivere su ogni argomento concepibile e come per lui, essendo poeta, la parola scritta fosse in grado di includere ogni virtuosismo visivo. Si può leggere in questa luce il seguente madrigale, ovrero il ritratto di Aurelio Orsi, in cui la scrittura è considerata come eternatrice e superiore alle arti plastiche perché altrettanto potente del metallo e del marmo: 
Fu scarpel la mia penna,

e le carte, ov'incisi etermi carmi,

carte non fur, ma fur metalli, e marmi.

non intagliò già mai Scultor Latino

pari al mio stil dirino

idoli vivi e simulacri veri

ne' cori e ne' pensieri.

Quindi Apollo m'accenna

che quel c'ho sculto in sì leggiadri modi

lodato fia mentre vivan le lodi (Marino 1979, 174, corsivo nostro).

Quale posizione prende Marino sul parallelo tra pittura e poesia? Marino sostiene che la pittura non riesce sempre a cogliere l'essenza più misteriosa del rappresentato. A tal proposito, nella "Diceria Prima" sulla pittura, inclusa nelle Dicerie sacre, Marino si esprime così:

La diligenza, ne' pittori mortali, è fallace percioché per molto diligenti e sofferenti ch'essi sieno, non possono tanti riguardi avere che in qualche cosa non manchino, o che l'opera appieno corrisponda alla volontà, sí che in essa si ammiri perfettamente la maestria del disegno posseduto con sicurezza e maneggiato con pratica, la freschezza del colorito esprimere negli atti esteriori gli affetti interni dell'animo (Narino 1960, 93).

La relazione tra poesia e pittura viene intesa da Marino come un tentativo di sfidare moduli espressivi tradizionali riferiti ad un unico linguaggio, letterario o artistico. Ma nella Galeria il problema della corrispondenza delle arti sorelle non viene risolto. In genere, anche se non necessariamente, l'ekphrasis si riferisce a come la poesia interpreta le arti, e in modo specifico la pittura. La parola è in riferimento a delle immagini, ma perché vi sia ekphrasis queste non devono necessariamente essere opere d'arte. Si è anche visto come l'immagine possa esistere o meno, perché quello che importa è che il testo vi faccia riferimento in senso reale o ideale. Per Marino si può parlare, con certi limiti, sia di ekphrasis nozionale, orvero quando un testo descrive un'immagine che non ha realtà al di fuori di quel testo, sia di ekphrasis effettiva, che si ha quando l'immagine è indipendente dal testo. $\mathrm{Vi}$ è poi un terzo tipo di ekphrasis, cioè quella statica, che si ha quando il testo si riferisce a un solo momento dell'azione rappresentata nell'opera d'arte. Queste distinzioni sono di molta importanza nell'opera di Marino. Anche se Marino a volte afferma implicitamente una superiorità della poesia sulle altre arti, nelle già menzionate Dicerie sacre ripropone il concetto oraziano di ut pictura poesis senza toni polemici, affermando che pittura e poesia hanno molto in comune: 
Son tante le proporzioni e sí grandi l'analogie ch'al credere di tutti i savi passano tra le tele e le carte, tra i colori e gl'inchiostri, tra i pennelli e le penne, e somigliansi tanto queste due care gemelle nate d'un parto, dico pittura e poesia, che non è chi sappia giudicarle diverse: anzi tra se stesse le proprie qualità accumunando, ed insieme gli offici tutti e gli effetti confondendo, da chiunque ben le considera si possono quasi distinguere appena: la poesia è detta pittura parlante, la pittura poesia taciturna; dell'una è propria una mutola facondia, dell'altra un eloquente silenzio; questa tace in quella e quella ragiona in questa, onde scambiandosi alle volte reciprocamente la proprietà delle voci, la poesia dicesi dipignere $\mathrm{e}$ la pittura descrivere. Sono amendue ad un medesimo fine intente, cioè a pascere dilettevolmente gli animi umani e con sommo piacere consolargli, né altra differenza ha fra loro, se non che l'una imita con colori, l'altra con parole; l'una imita principalmente il di fuori, cioè le fattezze del corpo, l'altra il di dentro, cioè gli affetti dell'animo; l'una fa quasi intendere co' sensi, l'altra sentire con l'intelletto; l'una è intelleggibile ad ogni qualità di persone, eziandio ignoranti, l'altra non si lascia intendere se non da coloro che hanno studio e scienzia (Marino 1960, 151).

Dunque, per Marino pittura e poesia sembrerebbero essere intercambiabili, o almeno questo è ciò che si ricava dal passaggio appena citato. $\mathrm{Ma}$ questo accade solo perché Marino, con una di quelle antitesi di cui è ricca la sua poesia, riesce ad operare uno scambio di voci e di intenti, e ad invertire la funzione della poesia, cioè di descrivere, con quella del dipingere della pittura. Dal testo sopracitato si è visto che la poesia è "pittura parlante; mutola facondia; tace nella pittura" e mentre quest'ultima è "poesia taciturna; eloquente silenzio; ragiona nella poesia". L'intento delle due arti è quello di "pascer diletterolmente": la pittura "imita con colori; imita il di fuori, fa intendere co' sensi; [è] intelleggibile a tutti"; la poesia "imita con le parole, imita il di dentro, fa sentire con l'intelletto, [è] intelleggibile a chi sa". La pittura è spesso definita muta poesia, e qui forse Marino ha in mente (ma senza condividerle) le teorie di Leonardo, quando costui riafferma la superiorità della pittura, ovvero "diremo adonque la poesia essere scienza che sommamente opera nelli orbi, e la pittura far il medesimo nelli sordi, ma tanto resta più degna la pittura, quanto ella serve a miglior senso" (Barocchi 237) $)^{5}$.

Ciò che importa a Marino è che la pittura è del presente e descrive ciò che è "di fuori", mentre la poesia narra il passato e ciò che è "di dentro". Gli occhi devono possedere momento per momento. Ma ciò che sta "nella carte" è posseduto per sempre. Nella poesia al Conte Ridolfo Campeggi, in cui viene richiesto un ritratto, Marino scrive infatti: 
... Non per altra cagion la bramo e cheggio

se non per posseder con gli occhi ancora

in tela il volto tuo, sì come ognora

l'anima in carte espressamente io veggio (Narino 1979, 194).

Sempre a proposito delle varie definizioni di pittura e poesia, sarà interessante considerare il canto sesto dell'Adone, dove vengono confermate le sopraddette definizioni. Nella stanza 51, descrivendo Venere che mostra a Adone le stanze del palazzo dell'arte, Marino scrive:

Da tutti quattro i lati in ogni parte il muro a varie immagini è dipinto. Ciò che favoleggiar l'antiche carte degli amori celesti, in esso è finto.

Gl'innamorati dei mirabil arte

v'ombreggio sì, che 'l ver da l'ombra è vinto

$\mathrm{e}$, benché tutti mute abbian le lingue,

il silenzio e " parlar vi si distingue (Marino 1988 vol. 1, 317, corsivo nostro).

Commentando il canto in questione, Giovanni Pozzi scrive che "nell'ambito del vastissimo tema del modo col quale la pittura esprima il moto ed il suono, è notevole la parte che il Marino faceva al silenzio come oggetto dell'espressione pittorica" (Marino 1988 vol. 2, 335). Basta infatti ricordare la seguente lirica sul Cornelio Musso di Bernardo Castello:

Tace, Bernardo, o parla

il gran Cornelio in tue vivaci carte?

Se parla, ond'è, che 'l suon de le sue note

udir altri non pote?

Se tace, or come fai

tacer chi a nostro pro non tacque mai?

O miracol de l'Arte!

Il silenzio è loquace,

la pittura eloquente, e parla, e tace (Marino 1979, 137).

Inoltre, dalla stanza 53 alla 57 del canto sesto dell' Adone segue una catalogazione di pittori più moderni di quelli di cui Marino parla nelle Dicerie sacre, e che nell' Adone vengono elencati secondo un criterio di appartenenza a scuole regionali. Dopo un'accurata elencazione di pittori, segue un commento alla descrizione riguardante gli amori degli dei, e soprattutto quelli riguardanti Giove (in particolare l'episodio della ninfa Europa), dalla stanza 58 alla 63 , introdotto nella 58 : 
A contemplar la loggia e la parete

il portier del giardino Adone invita,

Di mute poesie, d'istorie liete

imaginata tutta e colorita,

e del fanciul dal'arco e dala rete

i dolci effetti ad un ad un gli addita,

divisandogli a bocca or quelli, or questi

furtivi amori degli eroi celesti (Marino 1988 vol. 1, 319).

Marino, dunque, definisce i quadri come "mute poesie" a cui il poeta darà il suono con i giochi di parole, le paronomasie, e le altre tecniche stilistiche da lui adottate. Nei versi qui riportati, è da notare come Marino proponga una forma di ekphrasis nozionale e ideale, dato che il poeta fa riferimento a un'opera pittorica da lui inventata. Pozzi, a proposito delle stanze di cui si è detto, osserva che il raddoppiamento superlativale, (sembra e sembra; intorno intorno; trecce/ trecce; piano piano; tutta e tutta), le allitterazioni e le paronomasie, come nell'ottava 59 (schiera/verginelle/ scherza/ superta) e il riferimento finale all'eco facciano osservare come

...poco o nulla della descrizione mariniana rifletta $\mathrm{i}$ valori figurativi e plastici propri di una rappresentazione legata, come qui vuole il contesto, ad un'arte della vista: questa non è una 'storia' in pittura, ma è una storia narrata, tendente all'astrattezza del ritmo musicale (fatto doppiamente significativo nel contesto di un poema così poco narrativo e dalla parte di un uomo che scrisse la Galeria (Marino 1988 rol. 2, 338).

Come si ricorderà, Marzio Pieri, nella citata introduzione alla Galeria, aveva osservato che in questo modo, delle pitture, potrebbe parlare un cieco. Eugenia Paulicelli vi riscontra invece un desiderio di aggiungere alla dimensione rappresentativa "quella del mondo inventato, del sogno, del gioco infinito di specchi concavi, convessi, anamorfici della parola chiaroscurale, figurata, metamorfica" (Paulicelli 258).

Crediamo però che, più che di invenzione di nuove realtà, le poesie della Galeria abbiano a che fare con un problema di referenzialità che ripropone le sopracitate definizioni di ekphrasis in una luce completamente diversa. Marino supera le opere d'arte da lui descritte attraverso una nondescrizione in cui il ritratto verbale sostituisce quello pittorico, e non si tratta più di semplice illustrazione del visivo, ma di creazione che avviene insieme alla o prima della scrittura stessa. Spesso viene a mancare la referenzialità del testo all'opera che il poeta descrive o inventa. Ma molte poesie si trasformano così in una lode all'artista più che in una illustrazione verbale dell'opera pittorica a cui il poeta fa riferimento (e uno dei motivi è che, come già si è accennato, queste opere erano spesso una merce di 
scambio con i dipinti o disegni a cui si riferivano, ed ovviamente il tutto era finalizzato ad arricchire la collezione d'arte di Marino). ${ }^{6}$

Però, se il compito dell'ekphrasis è anche quello di dare voce alle immagini, sia che queste esistano o meno, Marino nelle sue poesie dà effettivamente voce ai soggetti da lui descritti, e contemporaneamente trasforma lo statuto della parola. Viene ribadita la superiorità della parola scritta sull'immagine e, pertanto, scrive così la Paulicelli:

Allora la corrispondenza sarà da ricercarsi su altri versanti, come e se la parola nell'ambito della Galeria venga di fatto a sostituirsi all'effetto mimetico della pittura rispetto all'oggetto che riproduce o rappresenta; o a raggiungere, attraverso i dispositivi retorici dell'arguzia, quella energeia che consente alla parola di diventare visiva, tattile (Paulicelli 259).

A questo proposito, una lirica in cui Marino afferma chiaramente tale principio è il suo ritratto di Ludovico Ariosto:

Quel gran Pittor de l'armi e degli amori, di Pindo unico Sol, canoro mostro del Re de' fiumi, e Re degli Scrittori, or qui dipinto a gli occhi altrui mi mostro.

Ma meglio che i pennelli e che i colori la mia penna dipingono, e 'l mio inchiostro.

Più viva la mia immagine, o Pittori esprime il libro mio, che 'l quadro vostro.

Caduche sono le vostre tele, eterne le carte mie. Voi solo il corpo, ed io dipingo sì che l'anima si scerne.

A dipinger non prenda il volto mio chi dipinger non sa tra le superne maraviglie del Ciel Natura, e Dio (Marino 1979, 176).

In questo sonetto, l'Ariosto di Marino è il Re degli Scrittori ed è paragonato all'inizio al Pittor de l'armi e degli amori, che è Tiziano. Ma il ritratto mariniano di Ariosto parla in prima persona, dando quindi vita ad un immaginario autoritratto. Secondo la Paulicelli, "il ricorrere della parola dipingere, [è] usat[o] volutamente come metafora della scrittura poetica; con essa si realizza a livello formale una funzione icastica della parola" (Paulicelli 261), anche se, secondo noi, qui si ha soprattutto una vera e propria dichiarazione di poetica da parte dell'autore. La pittura continua a mancare in qualche cosa, anche se il disegno ben realizzato riesce ad esprimere i moti dell'animo. A questo proposito, Marino, in Sopra il ritratto della sua Donna. Ad Ambrogio Figino, scrive: 
Ben può, Figin, de la tua nobil mano lo stil certo divin, l'arte celeste

l'alte bellezze e le sembianze oneste formar de l'Idol mio sommo e sovrano.

Ma que' lumi ombreggiar presume invano, che quasi gemme in lucid'òr conteste nasconde in lui la corrottibil veste, con terreni colori ingegno umano.

Può ben uom de la neve il bel candore e del foco il vermiglio in tela espresso ritrar, ma non il gelo, e non l'ardore; e la forma imitar del Sole istesso: ma 'l moto e la virtì del suo splendore in pittura mostrar non è concesso (Marino 1979, 249-50, corsivo nostro).

L'immortalità artistica è riservata soprattutto al poeta, e quindi sono eterne le sue carte, in cui si trova una nobile illustrazione dell'anima, mentre al pittore è riservata la descrizione del corpo umano, ovvero "la corrottibil veste" dipinta "con terreni colori". Inoltre, le immagini poetiche sono più vive che nei quadri, forse perché la parola scritta dà la possibilità alla fantasia di creare dei mondi immaginari in cui la meraviglia e la sorpresa non sono vincolate ai limiti della tecnica pittorica. A ciò si può aggiungere che per Marino la poesia, al contrario della pittura, può imitare "moto" e "virtu". La poesia si presenta dunque come un'arte in cui lo spazio è soggetto al tempo, al movimento e a un rapporto dinamico con crescita e diminuzione. In nuce è come se fosse già presente il criterio della distinzione tra pittura come arte dello spazio e poesia come arte del tempo, distinzione che sarà affermata da Lessing nel suo saggio sul Laocoonte nella seconda metà del Settecento.

Non sempre Marino interviene con molti commenti riguardanti l'opera pittorica da lui descritta o, meglio, narrata. Un esempio di questo è la poesia che segue, ovvero Endimione, che riguarda la Luna di Carlo Viniziano:

Sotto il freddo seren su l'erba assiso presso il fidato Can, lungo l'armento stavasi Endimion col guardo intento il suo notturno Sol mirando fiso.

E con selvaggio e rustico sorriso l'ombra additando del macchiato argento, notava sol fra cento raggi e cento le stampe de' suoi baci entro il bel viso. 
E la scorgea per mezzo il folto orrore

rotar il carro lampeggiante e vago

rossa di scorno, e pallida d'amore.

Quando Carlo il ritrasse, e del suo Vago

correndo Cinthia al dilettoso errore,

rifiutò 'I vero, ed abbracciò l'imago (Marino 1979, 16).

Qui nulla interferisce con la descrizione verbale del quadro, e la lirica si fa completamente visiva. La rappresentazione è cosi bella che persino la luna preferisce, di Endimione, l'immagine al reale. Ma l"'imago" di cui qui parla Marino non è l'immagine pittorica, bensì l'immaginazione contrapposta vittoriosamente al vero. Secondo la tradizione rinascimentale e tardo-rinascimentale seguita da Marino, tutta l'arte trionfa sulla natura, la poesia come la pittura. Cosi si può leggere, ad esempio, nella stanza XIV dei versi dedicati alla Maddalena di Tiziano:

Ma ceda la natura e ceda il vero

a quel che dotto artefice ne finse,

ché, qual l'avea ne l'alma e nel pensiero

tal bella e viva ancor qui la dipinse.

Oh celeste sembianza, oh magistero,

ove ne l'opra sua se stesso ei vinse;

fregio eterno de' lini e de le carte,

meraviglia del mondo, onor de l'arte (Marino 1979, 74).

La pittura, prodotta da un "dotto artefice" è finzione ("ne finse") di moti d'animo dell'artista ("qual l'avea ne l'alma e nel pensier"), ma è finzione a cui il vero deve "cedere", anche perché l'opera pur fingendo coglie un attimo di vita del soggetto ritratto ("tal bella e viva ancora qui la dipinse"). E la maestria di Tiziano è però tale che Marino dice che il pittore ha vinto se stesso creando un fregio eterno, meraviglia del mondo e onore dell'arte, lode che di solito riserva principalmente alla poesia.

Il tema della natura che viene ri-creata dall'artista, il quale deve superarla, costituisce il tema fondamentale del canto nono dell'Adone, con la descrizione del palazzo di Venere, che rappresenta il trionfo dell'Arte sulla Natura. Guglielminetti fa notare come la struttura dell'edificio non sia circolare e labirintica come quello in cui abita l'Armida di Tasso, ma abbia quattro torri che lo circondano e una struttura quadrata, come si legge nell'Adone IX, 94: "in mezzo al quadrangolo a misura/la pianta de la fabrica si posa". Il palazzo è formato da un quadrato di portici, ed ha un numero corrispondente di giardini e una torre rotonda in mezzo, mentre ogni angolo ha un giardino ed una torre. Nel contesto dell'Adone, scrive Martini: 
il capriccio in senso lato, dominando il collezionismo esoterico dei canti della conoscenza intellettiva (10-11), si contrappone alla simmetria della vasta fabbrica del giardino (canti 6-8), dove avviene la conoscenza sensitiva e dove Pittura, Musica e Poesia hanno la loro regolare sede: ultima sorpresa offertaci da un poeta che non fu, come il Lomazzo, adoratore del caos, ma certo un laborioso e avveduto aggiratore del nulla, e sentì come pochi l'originale raccapriccio del vuoto" (Martini 664).

Questo vuoto è riempito completamente, e in questi spazi occupati da produzioni artistiche si coglie l'aspetto più scenografico del loro ordine. Guglielminetti osserva come ci siano "elementi tipici della conosciuta ricchezza scenografica del palcoscenico barocco: la «fusione di spazi» cioè, gli «assi spaziali sviluppantisi in profondità», la «ricchezza di movimenti delle arti del cambiamento di scena)" (Guglielminetti 123) e c'è fluidità in questi movimenti di scena in scena. La descrizione del palazzo di Venere va dal canto sesto al nono, e quest'ultimo è dedicato alla poesia e all'iniziazione di Adone all'Arte.

Nella Galeria, la stessa tematica si ritrova anche nel testo In morte di Michelagnolo da Caravaggio:

Fecer crudel congiura,

Michele, a danni tuoi Morte, e Natura.

questa restar temea

de la tua mano in ogni imagin vinta,

ch'era da te creata e non dipinta.

Quella di sdegno arda,

perché con larga usura

quante la falce sua genti struggea,

tante il pennello tuo ne rifacea (Marino 1979, 191).

Un altro dipinto che, secondo Marino, è un esempio di come il pittore crei una nuova realtà nel rappresentato è La strage de' fanciulli innocenti di Guido Reni:

Che fai Guido? che fai?

La man, che forme angeliche dipigne, tratta or opre sanguigne?

Non vedi tu, che mentre il sanguignoso

stuol de' fanciulli ravivando vai,

nova morte gli dài?

O ne la crudeltate anco pietoso

Fabro gentil, ben sai

ch'ancor Tragico caso è caro oggetto,

e che spesso l'orror va col diletto (Marino 1979, 56). 
Questa poesia sarebbe doruta apparire nelle Rime, ma il poeta tolse da questo volume alcune liriche aventi come soggetto figure bibliche significative della storia di Gerusalemme (Colombo 64). In questo trattamento della strage degli innocenti, Marino loda la capacità del visivo di colpire gli altri sensi con il realismo del rappresentato e di creare compiacimento estetico anche da soggetti poco piacevoli, come un massacro, e inoltre di stupire con paradossi, come quello di dipingere figure angeliche nel contesto di opere sanguigne. Marino è colpito dalla veridicità di ciò che il quadro rappresenta. Sembra che alla morte arvenuta nella realtà segua un altro decesso fittizio ("nova morte gli dai"), e comunque il piacere che si prova guardando il quadro è dovuto alla constatazione molto "barocca" "che spesso l'orror va col diletto".

Il punto di vista di Marino rispetto al problema della gerarchia delle arti è ambiguo, ma nella sua ricorrente mancanza di una presa di posizione netta è anche ricco ed interessante. Il poeta napoletano afferma spesso come la poesia sia superiore alla pittura per una maggiore capacità di descrizione degli affetti, ma il realtà non pone delle rigide divisioni tra le due arti, che per lui spesso si equivalgono, come si vede dalla sua ammirazione incondizionata, ad esempio, per Tiziano, Cararaggio, ed altri grandi pittori dell'epoca. Inoltre, per quanto riguarda la questione dell'ekphrasis, Marino tenta di ricreare foneticamente, attraverso la poesia, la storia narrata nell'opera d'arte descritta. Cosi, le sue poesie ispirate alla pittura non sono mai delle pure e semplici descrizioni, ma tendono sempre verso la narrazione e ribadiscono quindi l'indipendenza della poesia rispetto alla pittura.

Rutgers University

\section{NOTE}

${ }^{1}$ Blakeney 36 , trad. nostra. Si legga anche la traduzione di Dorsch, orvero: "A poem is like a painting: the closer you stand to this one the more it will impress you, whereas you have to stand a good distance from that one; this one demands a rather dark corner, but that one needs to be seen in full light, and will stand up to the keen-eyed scrutiny of the art-critic; this one only pleased you the first time you saw it, but that one will go on giving pleasure however often it is looked at" (Dorsch 91-2). A titolo di curiosita si possono citare i versi che Charles Alfonse Du Fresnoy incluse nel suo poema latino De Arte Grapbica (scritto nel ] 637 e pubblicato nel 1667), e nel quale vengono argutamente combinate le posizioni di Orazio e quelle di Simonide: 
Lt pictura poesis erit; similisque poesi

Sit pictura: refert per aemula quaque sororem,

Alternantque vices et nomina: muta Poesis

Dicitur haec, Pictura loquens soleat illa vocari.

[Sia la poesia come la pittura; e la pittura rassomigli

la poesia: che competano l'un l'altra

e che alternino compiti e nomi: muta poesia

si chiami l'una, pittura parlante sarà chiamata l'altra.] (trad. nostra)

Testo latino citato in Markiewicz 537. Si veda anche Maiorino.

${ }^{2}$ Roger Simon commenta ancora sull'importanza della pittura per Marino, quando scrive: "Dans toute l'oeuvre de [Marino], la vision picturale joue un rôle primordial dans la création littéraire et ... l'oeuvre écrite peut avoir été génératrice de nouvelles créations artistiques" (Simon 1988a, 581). Dello stesso critico, si veda Simon 1988b. Sul tema delle immagini e del concetto in G.B. Marino, si veda Graziani.

${ }^{3}$ Sull'amicizia tra Marino e Poussin, si veda Ackerman.

${ }^{4} \mathrm{Si}$ reda il confronto tra il poeta inglese Marvell e Marino, per cui "Marino's largely literal gallery has been subtilized into a psychological metaphor; Marvell's gallery is in the soul", Jean Hagstrum 114.

${ }^{5} \mathrm{Ma}$ sul tema della superiorità della scrittura si veda anche Nemerow-Ulman.

${ }^{6} \mathrm{Si}$ reda in merito Mirollo.

\section{OPERE CITATE}

Ackerman, Gerard. "Giambattista Marino's Contribution to Seicento Art Theory", in The Art Bulletin, vol. XLIII, December 1961, pp. 326-336.

Barocchi, Paola (a cura di). Scritti d'arte del cinquecento. Vol. 1. Milano-Napoli: Ricciardi Editore, 1971.

Besomi, Ottario. "Fra i ritratti del Giovio e del Marino. Schede per la "Galeria"'.

Lettere Italiane. Anno XI, No. 4, Ottobre-Dicembre 1988, pp. 510-521.

Blakenes, Edward Henry (ed). Horace and the Art of Poetry. Oxford: University Press, 1928.

Colombo, Angelo. Ora l'armi scacciano le muse. Ricercbe su Gionan Battista Marino.

Università degli Studi di Parma. Centro Studî Archivio Barocco. Roma:

Archivio Guido Izzi, 1996

Dionisotti, Carlo. "La Galleria degli uomini illustri”. Lettere Italiane. Anno XXXI-

II, No. 4, Ottobre-Dicembre 1981, p. 482-492.

Dorsch, T.S. (ed). Classical Literary' Criticism. New York: Penguin, 1965.

Franceschetti, Antonio (a cura di). Letteratura italiana e arti figurative. Atti del XII

convegno dell'Associazione Internazionale per gli Studi di Lingua e Letteratura Italiana. Firenze: Olschki Editore, 1988. 
Golahny, Amy: "Ekphrasis in the Interarts Discourse", in Amy Golahny" (cd.) The Eye of the Poet: Studies in the Reciprocity of the I'isual and Literan' Arts from the Renaissance to the Present. London: Associated University Presses, 1996.

Graziani, I'rançoise. "L'image comme verbe divin chez Zuccaro et Marino." In: Actes du Congrès de la Société Française de Littérature Générale et Comparée. Art et Littérature. 1988; pp. 265-277.

Guardiani, Francesco (ed.) The Sense of Marino. New York-Ottawa-Toronto: Legas, 1994.

"L'idea dell'immagine nella Galeria di G.B. Marino". Antonio Franceschetti (a cura di). Letteratura italiana e arti figurative. Atti del XII conregno dell'Associazione Internazionale per gli Studi di Lingua e Letteratura Italiana. Firenze: Olschki Editore, 1988, pp. 647-654.

Guglielminetti, Marziano. Tecnica e imenzione nell'opera di Giambattista Marino. Messina-Firenze: Casa Editrice G. D'Anna, 1964.

Hagstrum, Jean. The Sister Arts. The Tradition of Literary Pictorialism and English Poetry from Dryden to Gray. Chicago: The University of Chicago Press, 1958.

Lee, Rensselaer. Ut Pictura Poesis: The Humanistic Theory of Painting. New York: Norton \& Company, Inc., 1967.

Maiorino, Giancarlo. The Cornucopian Mlind and the Baroque Lnity of the Arts. University Park-London: The Pennsylvania State L'niversity Press, 1990.

Marino, Gian Battista. Adone. A cura di Giovanni Pozzi. 2 Vol. Milano: Adelphi, 1988.

- Dicerie sacre e La strage de gl'Innocenti. A cura di Giovanni Pozzi. Torino, Einaudi, 196.

- La Galeria. 2 Vol. A cura di Marzio Pieri. Padora: Liviana Editrice, 1979. - Lettere e dedicatorie. A cura di Angelo Borzelli e Fausto Nicolini. Vol. 1. Bari: Laterza, 1911.

Markiewicz, Henryk. "Ut Pictura Poesis ... A History of the Topos and the Problem". New Literary History: A Journal of Theory and Interpretation, Vol. 18, Spring 1987, No. 3, pp. 535-558.

Martini, Alessandro. "I capricci del Marino tra pittura e musica", in Antonio Franceschetti, (a cura di). Letteratura italiana e arti figurative. Atti del XII convegno dell'Associazione Internazionale per gli Studi di Lingua e Letteratura Italiana. Firenze: Olschki Editore, 1988, pp. 655-664.

Mirollo, James. The Poet of the Marvelous. New York-London: Columbia L niversity Press, 1963.

Moses, Gavriel. 'Care Gemelle d'un Parto Nate: Marin"s Picta Poesis." Modern Langhage Notes, Italian Issue, Vol. 100, January 1985, No. 1, pp. 82-110.

Nemerow-Ulman, Linda. "Narrative Unities in Marino's I a Galeria", Italica, Vol. 64, n. 1, Spring 1987, pp. 76-86.

Paulicelli, Eugenia. "Parola e spazi visivi nella Galeria". In: Francesco Guardiani (ed.) The Sense of Marino. New York-Ottawa-Toronto: I egas, 1994, pp. 255-265. Praz, Mario. Mnemosyine: the Parallel between Literature and the I isnal Arts. The 1. IW. Mellon Lectures in the Fine Arts (1967) at the National Gallery of Art in Washington, D.C. Princeton: Princeton U'niversity Press, 1970. 
Simon, Roger. "Vision picturale et création poétique chez G.B. Marino." In: Actes du Congrès de la Société Française de Littérature Générale et Comparée. Art et Littérature, 1988, pp. 581-592

. "Notes sur quelques lectures de G.B. Marino." In: Actes du Congrès de la Société Française de Littérature Générale et Comparée. Art et Littérature, 1988; pp. 51-68

Tatarkiewicz, Wladyslaw. A History of Six Ideas: An Essay in Aestbetics. The Hague: Martinus Nijhoff, 1980

Verdicchio, Massimo. “Ut Pictura Poesis' o le corna di Lessing”. pp. 773-781; in: Antonio Franceschetti (a cura di). Letteratura italiana e arti figurative. Atti del XII convegno dell'Associazione Internazionale per gli Studi di Lingua e Letteratura Italiana. Firenze: Olschki Editore, 1988

Warman, Stephen. "The Subject-Matter and Treatment of Marino's Images". Studi secenteschi. Vol. X, 1969 , pp. 57-131 